\title{
Tribological Characteristics of Manufactured Carbon Under Extreme Contact Conditions
}

\author{
E. Ross ${ }^{a}, *$, A. Ignatova, P. Stoyanov ${ }^{a}$ \\ ${ }^{a}$ Materials and Processes Engineering, Pratt \& Whitney, a division of Raytheon Technologies Corporation, East \\ Hartford, CT, USA.
}

Keywords:

Aerospace tribology

Graphite

Thermal spray

Tribological coatings

Third bodies

* Corresponding author:

Eli Ross

E-mail: eli.ross@prattwhitney.com

Received: 25 September 2020

Revised: 10 November 2020

Accepted: 22 June 2021

\begin{abstract}
A B S T R A C T
The purpose of this study was to investigate the influence of contact pressure on the friction and wear behavior of manufactured carbon. The ultimate goal was to identify the friction coefficient behavior of these systems as well as establish an understanding of the interfacial processes driving the frictional response. Experiments were conducted using a custom-build test apparatus with manufactured carbon running against chromium carbide thermal spray-coated low alloy steel substrates in the unlubricated condition. The results showed that the measured coefficient of friction for this tribocouple decreases with increasing contact pressure. The wear behavior of the manufactured carbon was found to be inversely proportional to the friction coefficient observations, with the highest contact pressure condition resulting in high wear. In addition, ex situ high magnification visual and cross-sectional analysis was performed in order to capture transfer film and other related phenomena and understand the difference in relative friction and wear response.
\end{abstract}

(C) 2021 Published by Faculty of Engineering

\section{INTRODUCTION}

Materials development and performance improvement of tribological systems in demanding applications such as gas turbine engines requires extensive testing as well as high fidelity ex situ analysis [1-5]. Examples of classes of tribological materials used in gas turbine engine have been previously reported [1,5] and are shown in Figure 1. In general, such tribological materials in gas turbine engines are classified into three categories: 1 ) hard coatings, 2) soft coatings, and 3) solid lubricants.

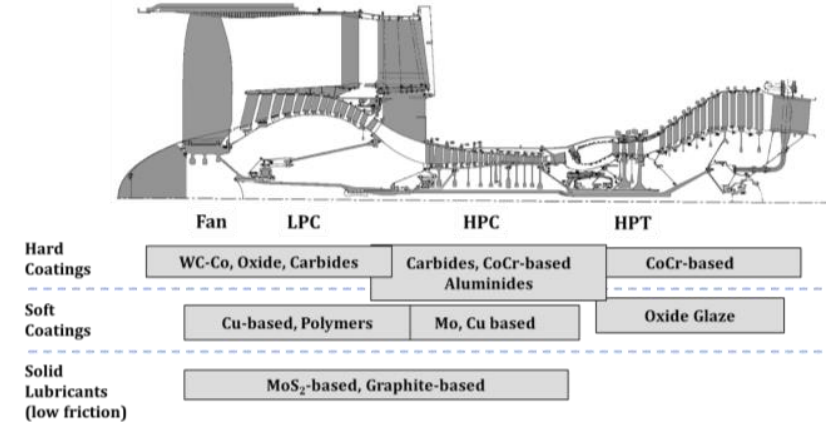

Fig. 1. Example of contact locations and tribological materials used in jet engine [5]. 
Carbon-based materials, either as surface treatments or monolithic materials, are commonly used on tribological interfaces in the fan, low and high pressure compressor regions of the gas turbine engine with the purpose to improve the wear resistance of certain components [1, 5-9] with minimal kinematic efficiency losses due to friction or associated heat generation. Such manufactured carbons which normally refer to monolithic materials comprised of nongraphitic and/or graphitic forms of carbon [9] are commonly used against hard coatings as shown with the examples in Figure 1. While some research has been previously performed on the manufacturing process [10] and mechanical properties [10] of the different carbon grades, there is limited research on the tribological performance of such systems under extreme conditions.

The purpose of this study is to provide a better understanding of the friction and wear behavior of manufactured carbons under aggressive contact conditions as well as understand the influence of contact pressure and sliding velocities on their tribological behavior. This requires a systematic study of 1) the friction and wear performance of manufactured carbon and 2) the interfacial mechanisms governing the friction and wear behavior, including mechanical mixing, transfer films and microstructural changes.

\section{EXPERIMENTAL PROCEDURE}

In this study, flat-on-flat experiments were conducted using a custom-built high temperature tribology apparatus. More specifically, the carbon element was in the form of a stationary ring while the rotating counterface was a chromium-carbide coated steel ring. The carbon used in this study is in the form of engineered carbon graphite. More details on the manufacturing process, structure and properties of such materials can be found here [11]. The chromium carbide coating was deposited on the low alloy steel substrates by means of flame spray deposition. More information on the deposition process and typical material characteristics for similar coatings can be found here [12]. Both, the disc and ring used in this study have a sealing diameter of $100 \mathrm{~mm}$. The experiments in this study were performed using two different normal forces of $50 \mathrm{~N}$ ( $\mathrm{P}_{\text {low }}$ ) and $75 \mathrm{~N}$ $\left(\mathrm{P}_{\text {high }}\right)$, with a relative surface velocity of $\mathrm{V}_{\text {normalized }}=$
1.25 , where $V_{\text {normalized }}$ is defined as the relative sliding velocity as compared with velocities typical of gas turbine engine sealing applications. Two tests were performed for each condition. The contact pressure is comprised of both mechanical and air pressure (differential) components. The experiments were performed at $200^{\circ} \mathrm{C}$ and the friction values were normalized, where the generated data has been divided by taking the ratio of the actual (i.e. measured) coefficient of friction at the reference temperature to the typical friction coefficient value of this system at room temperature. Similarly, the wear data presented in this study was normalized with respect to typical wear rates experienced in gas turbine engine seal applications.

The sliding surfaces were polished down to an average roughness of $0.35 \mu \mathrm{m}$ or better. The wear measurements on both surfaces were taken ex situ using a WLI (white light interferometer). In order to provide a better understanding of the interfacial phenomena, $e x$ situ analysis of the worn surfaces was performed using an optical microscope as well as Raman microscopy and X-ray diffraction (XRD).

The Raman analysis were perfumed using a DXRxi (Thermo Fisher) microspectrometer, equipped with $\lambda=532 \mathrm{~nm}$ laser and grating providing spectral resolution $\sim 3 \mathrm{~cm}^{-1}$. Lateral resolution was better than $2 \mu \mathrm{m}$. The worn samples were mounted on motorized displacement stage $( \pm 0.1$ $\mu \mathrm{m})$ and 2D Raman maps were taken across the wear tracks. All Raman measurement were done with unpolarized light at room temperature. The raw maps were processed by Thermo software to derive metrics of interest and post processed by ImageJ to create the corresponding distributions over the mapped areas.

\section{RESULTS AND DISCUSSION}

\subsection{Coefficient of friction}

The friction behavior as a function of sliding cycles is shown in Figure 2 for the two different contact stress conditions. Upon initial sliding, the coefficient of friction is higher for the tests run at $\mathrm{P}_{\text {high }}$ conditions. However, within the first few cycles, the friction decreases at a fast rate down to approximately $50 \%$ of the value associated with $\mathrm{P}_{\text {low }}$ test conditions. Subsequently 
to this running-in phase, the average coefficient of friction for both conditions remain roughly steady for the remainder of the test period. For the specific conditions tested here, the results indicate that the steady state friction coefficient decreases with increasing contact pressure.

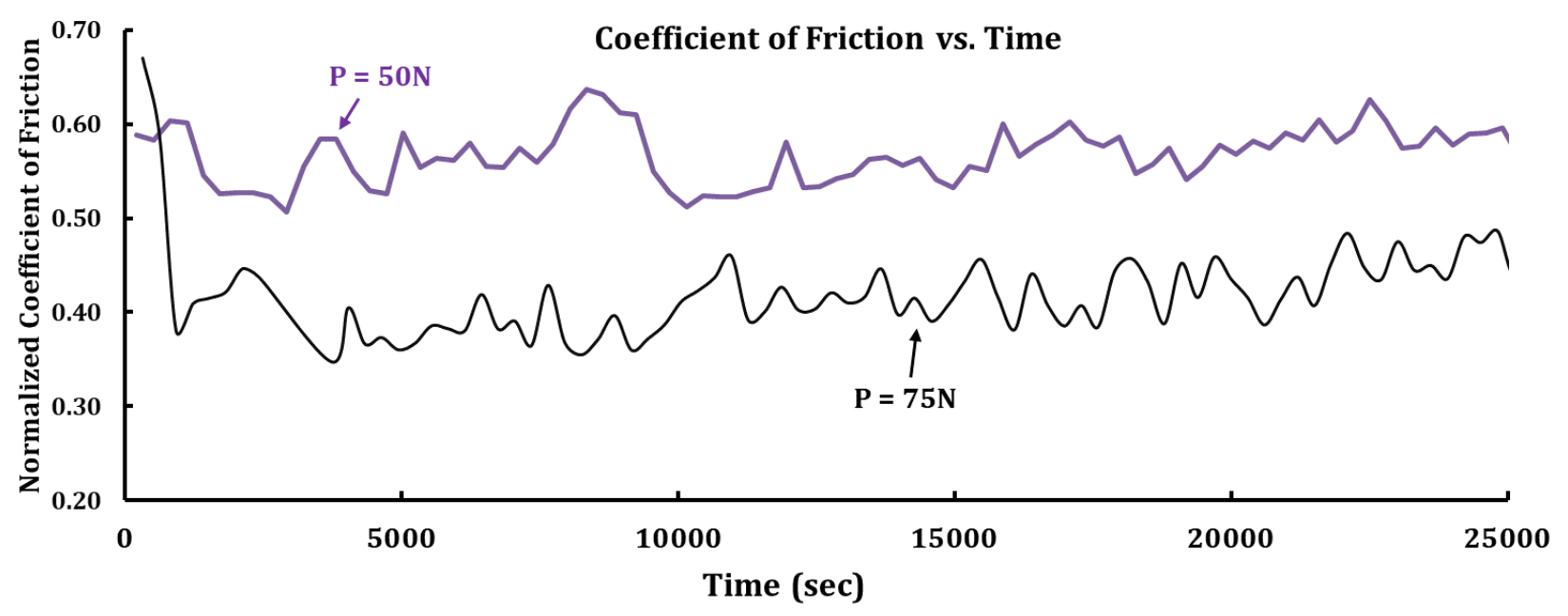

Fig. 2. Friction behavior for the testing performed using a normal load of $50 \mathrm{~N}$ and $75 \mathrm{~N}$.

\subsection{Wear behavior}

In contrast with the friction coefficient, the demonstrated carbon wear of the two contact conditions increased with increasing contact pressure, as shown in Figure 3 (i.e. measured at end of the test). Even accounting for test-to-test variation, the carbon wear is significantly higher for $\mathrm{P}_{\text {high }}$ tests. It should be noted no measurable wear of the counterface surface was detected for either test condition.

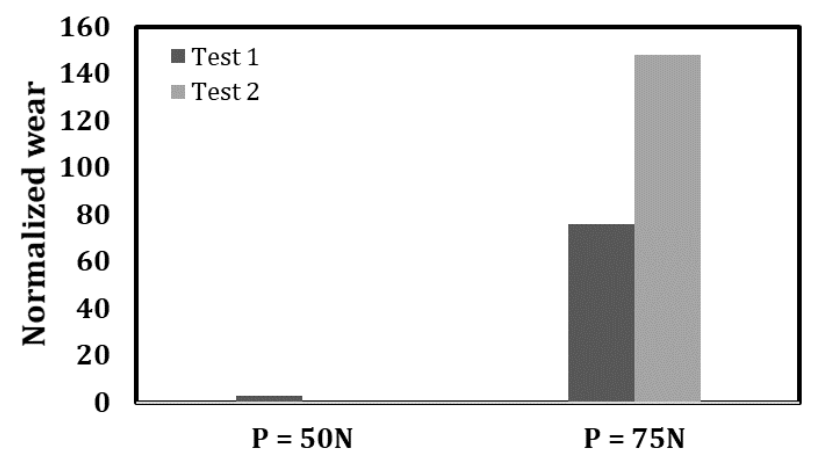

Fig. 3. Wear behavior vs normal load for the tests performed in this study.

\subsection{Ex situ analysis}

Ex situ analysis by means of optical microscopy and micro-Raman was performed in order to elucidate on the interfacial processes leading to observed friction and wear behavior. The optical images of the worn coated seal surfaces created under the two different conditions are shown in
Figure 4 (a) and (b). Initial observations of the higher magnification images from the worn surfaces indicate that slightly higher degree of transfer film formation from the carbon onto the counterface (e.g., indicated by the darker region on the wear track) associated with $\mathrm{P}_{\text {high }}$ condition. In addition, the transfer film on the samples tested at $P_{\text {high }}$ appear more uniform across the wear track, as well as showing localized micro-cracking of the counterface coating (Figure 4(b)).

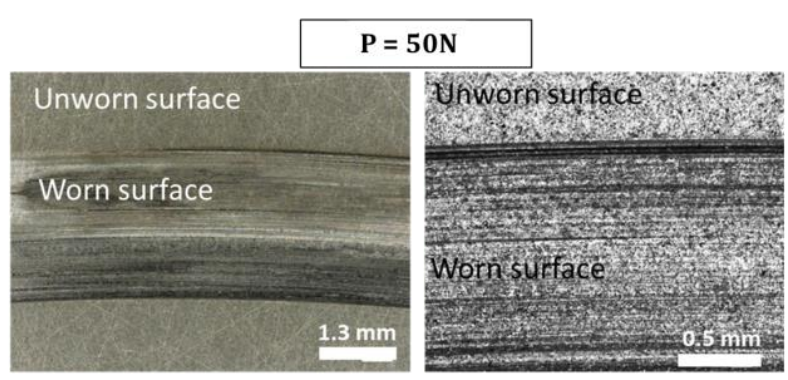

(a)

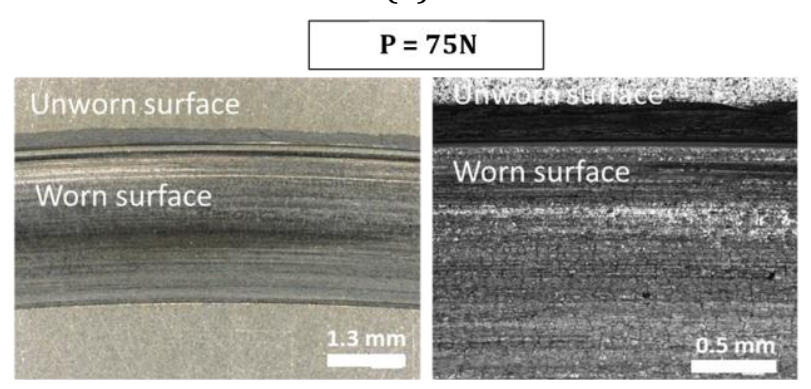

(b)

Fig. 4. Ex situ analysis of the worn counterfaces under (a)low contact stress and (b) high contact stress. 
Raman microscopy is a non-destructive technique which provides information at the micrometer scale regarding phase composition and crystalline state (i.e., form and structure of the carbon-based transfer film). Further analysis was performed using micro-Raman evaluation in order to better understand the transfer film phenomena. Figure 5 shows the Raman spectra obtained by averaging over 2D mapped regions in the worn surfaces of the $\mathrm{P}_{\text {low }}$ test conditions (red) and $\mathrm{P}_{\text {high }}$ test conditions (blue). The intensity of the two peaks is clearly higher for the $\mathrm{P}_{\text {high }}$ tests compared to the $\mathrm{P}_{\text {low }}$ tests. Notably, the apparent I(D)/I(G) ratio is slightly lower for the test performed under $\mathrm{P}_{\text {low. }}$ It should be noted that additional ex situ analysis was also performed by means of XRD (see Figure 6) and overall did not show any major differences between the worn and unworn surface. However, the worn surface created with the higher normal load (Plow) did show some evidence of $\mathrm{Cr}_{2} \mathrm{O}_{3}$.

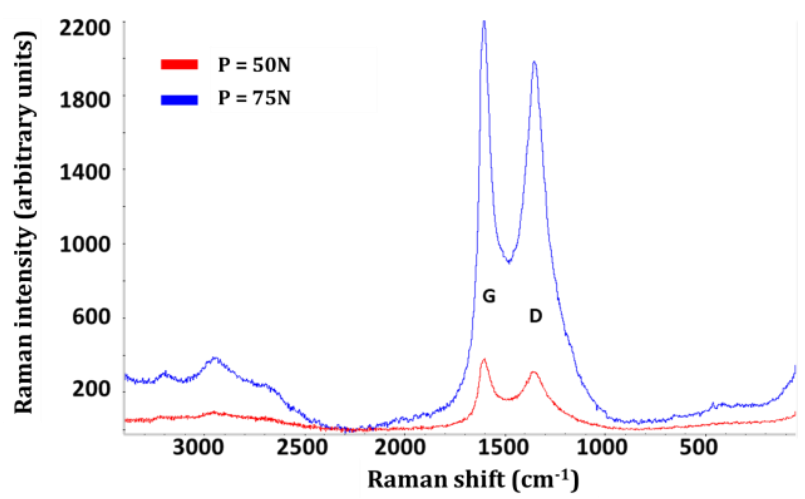

Fig. 5. Comparing Raman spectra obtained by averaging over 2D mapped areas: $\mathrm{P}_{\text {low }}$ (red), $\mathrm{P}_{\text {high }}$ (blue). Analyzed area is approximately $2500 \mu \mathrm{m} \times 100 \mu \mathrm{m}$ for each sample.

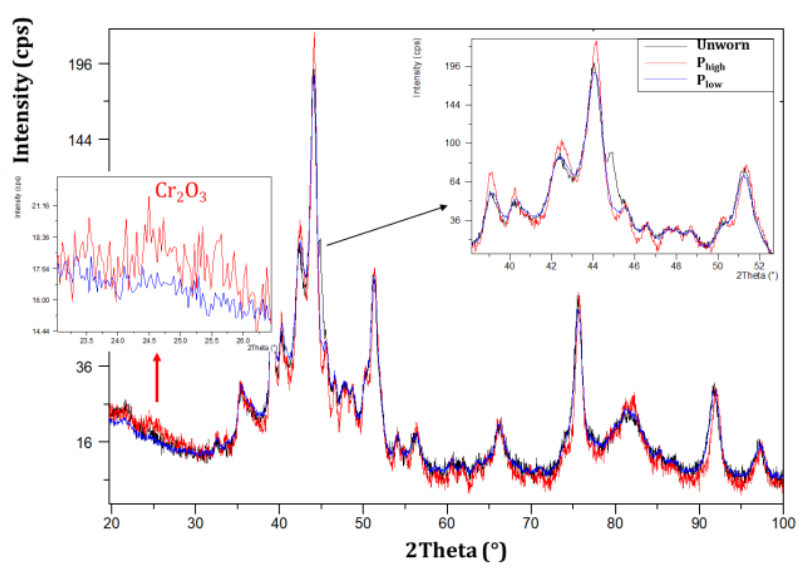

Fig. 6. XRD analysis of unworn and worn surfaces for the two conditions.

\subsection{Discussion}

The influence of contact pressure on the tribological performance of manufactured carbon was investigated in this study using a simple flaton-flat ring configuration. The results suggest that the coefficient of friction decreases with increasing contact pressure. This behavior has previously been observed, for example by Guo et al. [6] with resin impregnated carbon running against WC-Ni coated surfaces. When varying the load by a factor of five, the authors showed that the friction decreased for all sliding velocities and the different environments. Similarly, the decrease in friction coefficient has also been observed with other carbon based systems such as diamond-like carbon coatings [7], as well as other solid lubricants [8]. This behavior can typically be attributed to differences in the velocity accommodation modes and more specifically, the transfer film behavior.

Similarly, the ex-situ analysis of the wear tracks in this study revealed a higher amount of carbon transfer film on the experiments with the higher normal load $\left(\mathrm{P}_{\text {high }}\right)$. This was also consistent with the micro-Raman analysis, which showed a higher integrated peak areas for the tests run at $\mathrm{P}_{\text {high. }}$. The transfer film thickness can be related to the intensity of the Raman peaks $[13,14]$, which is consistent with the visual observations of the transfer film. It should be noted that intensity peak ratio $\mathrm{I}(\mathrm{D}) / \mathrm{I}(\mathrm{G})$ is typically used to determine structure, morphology and quality of graphitebased materials, with typically lower ratio being composed of higher graphite concentration [1517]. While there is a slight difference in the ratio between the two contact pressures in our study, it is within the statistical error. Additional work is currently being performed to better correlate this ratio to the wear and friction behavior of manufactured carbon.

The carbon wear behavior for the two contact conditions was substantially different, where the higher wear of the carbon is observed with the $\mathrm{P}_{\text {high }}$ condition. This correlates well with the exsitu analysis and in particular the higher degree of material transfer onto the counterface. The higher rate of carbon transfer is likely occurring mostly throughout the running-in phase for the tests with the higher contact pressure. This is observed with the decrease in friction coefficient within the first few cycles. The decrease in 
friction coefficient during the running-in phase has been previously reported with solid lubricants and attributed to mechanisms of initial tribofilm formation [14].

\section{CONCLUSION}

In this study we investigated the friction and wear behavior of manufactured carbon at two contact pressure conditions for a constant sliding speed. The results showed that the coefficient of friction for this tribocouple decreases with increasing normal load. In contrast, the wear behavior of carbon is inversely proportional to the friction coefficient observations, where the $\mathrm{P}_{\text {high }}$ condition resulted in higher wear. The higher wear for the $\mathrm{P}_{\text {high }}$ condition correlates well with the increased degree of carbon-based transfer film, which occurs likely during the first few cycles of the test. Further post-test visual examination and Raman microscopy was performed in order to evaluate the carbon transfer film phenomena which is believed to be the major contributor to the observed friction and wear behavior.

\section{REFERENCES}

[1] P. Stoyanov, K.M. Harrington, A. Frye, Insights into the Tribological Characteristic of Cu-Based Coatings Under Extreme Contact Conditions, JOM vol. 72, iss. 6, pp. 2191-2197, 2020, doi:10.1007/s11837-020-04087-7

[2] W. Smarsly, Coatings for advanced aero engine materials, in 8th High power impulse magnetron sputtering (HIPIMS) conference, 1-2 July, 2009, University of Sheffield, UK.

[3] R. Rajendran, Gas turbine coatings-An overview, Engineering Failure Analysis, vol. 26, pp. 355-369, 2012, doi: 10.1016/j.engfailanal.2012.07.007

[4] P. Stoyanov, L. Dawag, D.G. Goberman, D. Shah, Friction and Wear Characteristics of Single Crystal Ni-Based Superalloys at Elevated Temperatures, Tribology Letters, vol. 66, iss. 1, pp. 1-9, 2018, doi: 10.1007/s11249-018-0994-1

[5] P. Stoyanov, A. Wusatowska-Sarnek, T. Kasprow, Tribological Systems Solutions for Gas Turbine Engines, in International Conference on Metallurgical Coatings and Thin Films (ICMCTF), 24-28 April, 2018, San Diego, CA, USA.
[6] F. Guo, Y. Tian, Y. Liu, Y. Wang, Tribological behaviors of graphite sliding against cemented carbide in CaCl2 solution, Surface Topography: Metrology and Properties, vol. 3, no. 4, 2015, doi: 10.1088/2051-672X/3/4/044003

[7] T.W. Scharf, S.V Prasad, Solid lubricants: A review, Journal of Materials Science, vol. 48, pp. 511-531, 2012, doi: 10.1007/s10853-0127038-2

[8] P. Stoyanov, H.W. Strauss, R.R. Chromik, Scaling effects between micro-and macro-tribology for a Ti-MoS2 coating, Wear, vol. 274-275, pp. 149161, 2012, doi: 10.1016/j.wear.2011.08.021

[9] POCO Graphite Inc., Properties and Characterization of Graphite, Specialty Materials, For industrial applications, 2015.

[10] H.W. Davidson, Manufactured Carbon, Elsevier, 1968.

[11] Entegris Inc., Properties and characteristics of graphite, Specialty Materials, For the semiconductor industry, 2013.

[12] R.C. Tucker Jr, Plasma spray, detonation gun and hvof deposition techniques, in Y. Pauleau (Ed.): Materials and Processes for Surface and Interface Engineering, NATO ASI Series, vol. 290 , pp. 245-284, 2012

[13] T.W. Scharf, I.L. Singer, Quantification of the Thickness of Carbon Transfer Films Using Raman Tribometry, Tribology Letters, vol. 14, pp. 137145, 2003, doi: 10.1023/A:1021942822261

[14] P. Stoyanov, J. Schneider, M. Rinke, S. Ulrich, E. Nold, M. Dienwiebel, M. Stüber, Microstructure, mechanical properties and friction behavior of magnetron-sputtered $V$-C coatings, Surface and Coatings Technology, vol. 321, pp. 366-377, 2017, doi: 10.1016/j.surfcoat.2017.04.050

[15] N. Kumar, Load depended friction coefficient of crystalline graphite and anomalous behavior of wear dimensions, Tribology International, vol. 88, pp. 280-289, 2015, doi: 10.1016/j.triboint.2015.03.034

[16] A.C. Ferrari, Determination of bonding in diamond-like carbon by Raman spectroscopy, Diamond and Related Materials, vol. 11, iss. 3-6, pp. 1053-1061, 2002, doi: 10.1016/S09259635(01)00730-0

[17] A.C. Ferrari, J. Robertson, Resonant Raman spectroscopy of disordered, amorphous, and diamondlike carbon, Physical review B, vol. 64, iss. 7, pp. 1-13, 2001, doi: 10.1103/PhysRevB.64.075414 